\title{
Résumé des recommandations du Comité canadien sur l'immunisation concernant les programmes d'immunisation contre le virus du papillome humain
}

\author{
Comité canadien sur l'immunisation ${ }^{1 *}$ \\ *Acheminer toute correspondance par courriel au Secrétariat du CCI (cic-cci@phac-aspc.gc.ca)
}

\section{Contexte}

II existe plus d'une centaine de différents types de virus du papillome humain (VPH), et bon nombre d'entre eux ont été associés à des cancers et à des lésions bénignes du tractus anogénital, de la tête et du cou. Chez les femmes, presque tous les cancers du col de l'utérus peuvent être attribués à une infection par des types de VPH oncogènes, notamment les types 16 et 18 à risque élevé. Parmi les cancers liés au VPH chez les hommes, environ $92 \%$ des cancers de l'anus, $63 \%$ des cancers du pénis et $89 \%$ des cancers de la cavité buccale et des cancers de l'oropharynx sont attribuables aux types 16 et 18 du VPH à risque élevé. Les verrues anogénitales associées au VPH représentent également un fardeau économique et une diminution de la qualité de vie chez les hommes et les femmes. Des programmes d'immunisation des filles contre le VPH financés par les deniers publics sont actuellement en place dans toutes les provinces et tous les territoires; cependant, le taux de vaccination va de $60 \%$ à $85 \%$. En fonction des données scientifiques, le Comité consultatif national de l'immunisation (CCNI) recommande désormais l'immunisation contre le VPH chez les hommes et les femmes âgés de 9 à 26 ans pour la prévention de la maladie liée au VPH. En réponse aux indications élargies du vaccin quadrivalent contre le VPH et compte tenu de l'introduction d'un nouveau vaccin bivalent contre le VPH, le Comité canadien sur l'immunisation $(\mathrm{CCl})$ s'est penché sur les considérations liées aux programmes du vaccin contre le VPH et la pertinence de l'inclure dans les calendriers de vaccination systématique des provinces/territoires. Le présent article est un résumé des recommandations complètes du $\mathrm{CCl}$ en ce qui concerne le VPH (1).

\section{Objectif}

Formuler des recommandations concernant les programmes d'immunisation contre le VPH à la lumière de l'objectif du programme d'immunisation élargi contre le VPH, qui consiste à réduire la morbidité et la mortalité évitables liées au VPH dans la population canadienne.

\section{Approche}

Un groupe de travail du CCl s'est intéressé aux sujets suivants : caractéristiques et fardeau de la maladie, innocuité et efficacité du vaccin, faisabilité et acceptabilité, rentabilité, possibilité d'évaluer les programmes d'immunisation contre le VPH, questions liées à la recherche et considérations en matière d'équité et d'éthique.

\section{Observations}

Il existe actuellement deux vaccins contre le VPH dont l'utilisation est approuvée au Canada : Gardasil ${ }^{\mathrm{MD}}$ et Cervarix $^{\mathrm{MC}}$. Gardasil ${ }^{\mathrm{MD}}$, un vaccin quadrivalent, est approuvé chez les femmes âgées de 9 à 45 ans et les hommes âgés de 9 à 26 ans pour la prévention de l'infection causée par les types de VPH 6, 11, 16 et 18. Cervarix $^{\mathrm{MC}}$, un vaccin bivalent, est approuvé chez les femmes âgées de 9 à 25 ans pour la prévention du cancer du col de l'utérus causé par les types oncogènes du VPH 16 et 18. L'immunogénicité, l'efficacité et l'innocuité des deux vaccins ont été clairement démontrées; cependant, certaines études indiquent que l'immunogénicité et l'efficacité de la protection croisée du vaccin sont plus élevées pour Cervarix ${ }^{\mathrm{MC}}$. La protection quadrivalente du vaccin contre les verrues anogénitales s'est révélée plus importante sur le plan économique que les bienfaits potentiels du vaccin bivalent pour la prévention du cancer; par conséquent, le vaccin quadrivalent est plus rentable que le vaccin bivalent. 
Lorsque l'on prend en considération l'inclusion des hommes dans les programmes d'immunisation contre le VPH au Canada, les études sur le rapport coût/avantage indiquent qu'un programme pour les hommes devrait être rentable seulement lorsque la couverture vaccinale est inférieure à $50 \%$ parmi les femmes. Si un programme pour les hommes ciblant les garçons et les jeunes hommes à risque (p. ex. les hommes ayant des relations sexuelles avec d'autres hommes) était lancé, le vaccin quadrivalent serait le produit de choix, car c'est le seul dont l'utilisation chez les hommes est actuellement approuvée au Canada. Comme les provinces/territoires canadiens ont déjà mis en place des programmes d'immunisation contre le VPH pour les femmes, certaines évaluations ont été publiées. D'autres données s'imposent avant que l'on puisse procéder à une évaluation des nouveaux programmes de vaccination contre le VPH au niveau national.

De nombreux facteurs doivent être pris en compte lorsque l'on évalue les programmes d'immunisation contre le $\mathrm{VPH}$, tels que la présence de systèmes qui permettent de : 1) mesurer la couverture vaccinale et l'utilisation des vaccins, ainsi que la qualité des services d'immunisation; 2) mesurer les répercussions des infections liées au $\mathrm{VPH}$; 3) relier les bases de données sur les résultats pour la santé, les registres d'immunisation et les registres de la population. Les priorités en matière de recherche sur l'immunisation contre le VPH ont été déterminées dans le cadre de l'atelier national de 2005 sur les priorités de recherche concernant le VPH, la déclaration de 2012 du CCNI et le groupe d'experts sur le VPH, qui s'est réuni en juin 2013. Des indicateurs d'évaluation des répercussions de l'immunisation contre le VPH sur la population sont également en cours d'élaboration. Outre les considérations en matière d'équité et d'éthique, les répercussions de l'immunisation contre le VPH sur le dépistage du cancer du col de l'utérus devraient être examinées; un programme d'immunisation devrait faire partie intégrante d'un programme complet de prévention du cancer du col de l'utérus.

\section{Recommandations}

L'objectif du programme d'immunisation contre le VPH a été élargi en juin 2013; outre la réduction de la morbidité et de la mortalité liées au cancer du col de l'utérus, à ses précurseurs et aux autres cancers liés au VPH chez les femmes au Canada, il comprend désormais également la réduction de la morbidité et de la mortalité évitables liées au VPH dans la population canadienne dans son ensemble. L'objectif national élargi permet une certaine souplesse en ce qui concerne l'immunisation contre le VPH, grâce à laquelle on peut envisager d'autres aspects de la morbidité liée au VPH, ainsi que l'intégration des hommes et d'autres sous-groupes de la population aux programmes d'immunisation contre le VPH. De surcroît, il permet toujours de mettre l'accent sur la réduction de la morbidité et de la mortalité liées au cancer du col de l'utérus, à ses précurseurs et aux autres cancers liés au VPH chez les femmes.

À l'appui du nouvel objectif national, le $\mathrm{CCl}$ formule les recommandations suivantes : 1) améliorer les taux de couverture nationaux parmi les bénéficiaires du programme d'immunisation; 2) mettre la priorité sur l'évaluation et l'établissement d'indicateurs de programme; 3) traiter les priorités de recherche émergentes et non résolues; 4) intégrer de nouveaux groupes de population dans les programmes d'immunisation selon une démarche réfléchie basée sur les risques.

\section{Référence}

(1) Comité canadien sur l'immunisation. Recommandations relatives aux programmes de vaccination contre le virus du papillome humain. 2013.

http://publications.gc.ca/site/fra/464264/publication.html

${ }^{1}$ Remarque : Le Comité canadien sur l'immunisation offre des conseils d'ordre opérationnel et technique relatifs aux politiques et programmes d'immunisation au Canada. Il est composé de représentants des provinces/territoires, du Comité consultatif national de l'immunisation, de l'Advisory Committee on Immunization Practices (É.-U.) et de plusieurs instances de Santé Canada (Direction des produits biologiques et des thérapies génétiques; Direction des produits de santé commercialisés; Direction générale de la santé des Premières Nations et des Inuits). 